\title{
Exponential Synchronization of Neural Networks via Feedback Control in Complex Environment
}

\author{
Xiaoxiao Lv, ${ }^{1,2}$ Xiaodi Li $\mathbb{D},{ }^{1,3}$ Jinde Cao $\mathbb{D}^{2},{ }^{2}$ and Peiyong Duan ${ }^{4}$ \\ ${ }^{1}$ School of Mathematics and Statistics, Shandong Normal University, Jinan 250014, China \\ ${ }^{2}$ School of Mathematics, Southeast University, Nanjing 210096, China \\ ${ }^{3}$ Shandong Province Key Laboratory of Medical Physics and Image Processing Technology, Shandong Normal University, \\ Jinan, China \\ ${ }^{4}$ School of Information Science and Engineering, Shandong Normal University, Jinan 250014, China \\ Correspondence should be addressed to Xiaodi Li; 1xd@sdnu.edu.cn
}

Received 24 April 2018; Accepted 20 June 2018; Published 24 July 2018

Academic Editor: Andy Annamalai

Copyright (C) 2018 Xiaoxiao Lv et al. This is an open access article distributed under the Creative Commons Attribution License, which permits unrestricted use, distribution, and reproduction in any medium, provided the original work is properly cited.

\begin{abstract}
The problem of exponential synchronization for neural networks is investigated via feedback control in complex environment. By constructing suitable Lyapunov-Krasovskii functionals and applying the piecewise analytic method, some sufficient criteria for exponential synchronization of the addressed neural networks are established in terms of linear matrix inequalities (LMIs). The feedback control in complex environment includes the delayed aperiodically intermittent control and dynamic output feedback control. Moreover, the delayed aperiodically intermittent dynamic output feedback controller is designed based on the established LMIs. A numerical example and its numerical simulations are finally presented to show the effectiveness of obtained theoretical results.
\end{abstract}

\section{Introduction}

Neural networks have received significant attention during the past few decades due to their wide range of applications in different fields, such as signal processing, automatic control engineering, associative memory, parallel computing, combinatorial optimization, and pattern recognition [1-4]. In hardware implementation, time delays are inevitable due to the inherent information delivery time between neurons and the finite switching speed of amplifiers. The existence of time delays usually causes oscillation, divergence, or even instability of a system $[5,6]$, thus a large number of scholars have conducted a number of studies in regard to the dynamic behavior of the delayed neural networks [7-9]. From the perspective of time delays, all the research results can be divided into time-dependent and time-independent. The time-dependent results are usually less conservative than delay-independent ones, especially for systems with small delays. Therefore, many interesting results have been proposed in recent years, especially based on the Lyapunov-
Krasovskii functional method, linear matrix inequality (LMI) technique, $\mathbf{M}$ matrix approach, and so on [10-14]. On the other hand, synchronization is a typical collective behavior in nature, and it is observed in biological and physical systems, such as flocking of birds, synchronous glowing fireflies, wireless sensor networks, and synchronous transmissions of digital signals in communication networks $[15,16]$. The earliest research on synchronization can be traced back to the observation of the synchronous coupling phenomenon by C. Huygens in 1673. In general, synchronization means that a system is designed to simulate the dynamic behavior of another system, that is, the state trajectory of two systems is finally identical. Therefore, many important results on synchronization have been obtained in the last few years [17-20], and various control strategies have been developed to design effective controllers for achieving synchronization, such as adaptive control [21], pinning control [22], feedback control [23], impulsive control [24-27], and intermittent control [28]. 
As a kind of common continuous control, feedback control is widely applied to the system controller design problem. Actually, the feedback control can be divided into state feedback control [29], static output feedback control [30], and dynamic output feedback control [31]. It is well known that all the state variables of many practical systems cannot be measured directly, which means that the state feedback controller can be designed only if a state observer can be designed firstly and this will not only increase the cost but also decrease the system reliability. Therefore, the output feedback controller is more preferable when designing a controller to ensure the desired performance for the closed-loop system [32-34]. Different from continuous feedback control, intermittent control, as a kind of discontinuous feedback control strategy, consists of two parts: the nonzero control time and nonzero rest time. In particular, when the control time is reduced to zero, the intermittent control becomes the discontinuous impulsive control, and when the rest time is reduced to zero, it becomes continuous feedback control. Therefore, intermittent control combines the advantages of impulsive control and continuous feedback control. In the past decade, many valuable results have been obtained for synchronization of neural networks under intermittent control [35-39]. For example, by applying the average dwell time approach, structuring multiple Lyapunov-Krasovskii functions, and using Halanay inequality, new robust synchronization criteria are obtained for switched coupled networks via intermittent control [35]. In [36], the fast synchronization problem for a class of complex dynamical networks with time varying delay by means of periodically intermittent control was investigated. By designing appropriate adaptive intermittent controllers and using the Lyapunov stability theory, some pinning outer synchronization criteria are derived in [37], which can guarantee that the response network asymptotically synchronizes to the drive network.

Obviously, the above research results are periodic intermittent control strategies, which means that each control period and control time is fixed. Compared with the periodically intermittent control strategy, the aperiodically intermittent control has more flexibility and more applicability. Therefore, it is very meaningful to investigate the synchronization of neural networks under the aperiodically intermittent control. It is well known that the aperiodically intermittent control strategies were proposed by Liu and Chen in [40]. As shown in Figure 1, there is a time sequence $\zeta=\left\{t_{1}, t_{2}, \ldots\right\}$ satisfying $0=t_{1}<t_{2}<\cdots<t_{k}<\cdots$, $\lim _{k \rightarrow \infty} t_{k}=\infty$. The $k$ th time interval $\left[t_{k}, t_{k+1}\right), k \in \mathbb{Z}_{+}$is called the $k$ th control period and $t_{k+1}-t_{k}$ is called the $k$ th control period width. It can be seen clearly from Figure 1 that not all of the times in interval $\left[t_{k}, t_{k+1}\right)$ are controlled, among them, the interval $\left[t_{k}, t_{k}+\delta_{k}\right]$ is controlled, so $\left[t_{k}, t_{k}+\delta_{k}\right]$ is called the control time (or work time) and $\delta_{k}$ is called the $k$ th control width (control duration); while the interval $\left(t_{k}+\delta_{k}, t_{k+1}\right)$ is not controlled, so $\left(t_{k}+\delta_{k}, t_{k+1}\right)$ is called the rest time and $t_{k+1}-\left(t_{k}+\delta_{k}\right)$ is called the $k$ th rest width. Obviously, the control period and control width (or rest width) are both uncertain for different $k$ due to the aperiodically intermittent control strategy. In addition, once the control period and control width of every $k$ are

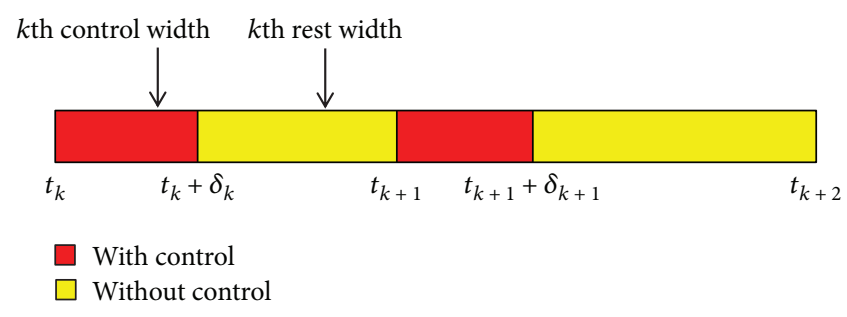

FIGURE 1: Sketch map of aperiodically intermittent control strategy.

fixed, that is, $t_{k+1}-t_{k}=T, \delta_{k}=\delta$, where $T>0$ and $\delta>0$ are constants, the aperiodically intermittent control strategy is transformed into the periodically intermittent control strategy, which has been considered in [41-43]. Due to the complexity of the control system, a single control method cannot effectively solve the poor performance of the system. In this case, the hybrid control method emerges at the historic moment, which makes the control system in complex environment. It has drawn researchers' widespread attention owing to the hybrid controller which combines the advantages of multiple individual controllers in complex environment. It is worth noting that the current attention for hybrid control strategy of synchronization of delayed neural networks is periodically intermittent dynamic output feedback control in complex environment. For example, the dynamic intermittent output feedback controller was designed to achieve exponential synchronization for master-slave neural networks [44]. However, there is no work that focuses on aperiodically intermittent dynamic output feedback control. These motivate the present study.

In this paper, we investigate the problems of exponential synchronization of neural networks via the feedback control in complex environment. By constructing suitable Lyapunov-Krasovskii functionals and applying the piecewise analytic method, some LMI-based sufficient conditions are established to guarantee the exponential synchronization of the addressed neural networks in terms of linear matrix inequalities (LMIs), which can be easily verified via the LMI toolbox. In particular, we develop the aperiodically intermittent dynamic output feedback control for synchronization of neural networks, consider the time delay in the design of the controller, and introduce the control rate of the control period, which in this sense are better than those results in [29-39]. The rest of this paper is organized as follows. In Section 2, some notations, a definition, and some well-known technical lemmas are given. Section 3 presents the criteria for exponential synchronization of neural networks and the design of the aperiodically intermittent dynamic output feedback controller. A numerical example is provided in Section 4 to demonstrate the effectiveness of the proposed criteria. Finally, the paper is concluded in Section 5.

\section{Preliminaries}

Notations. Let $\mathbb{R}$ denotes the set of real numbers, $\mathbb{R}_{+}$the set of positive numbers, $\mathbb{Z}_{+}$the set of positive integer, $\mathbb{N}$ 
the set of nonnegative integer, $\mathbb{R}^{n}$ the $n$-dimensional real spaces equipped with the Euclidean norm $|\bullet|$, and $\mathbb{R}^{n \times m}$ the $n \times m$-dimensional real spaces. $\mathbf{A}>0$ or $\mathbf{A}<0$ denotes that the matrix $\mathbf{A}$ is a symmetric and positive definite or negative definite matrix. The notation $\mathbf{A}^{T}$ and $\mathbf{A}^{-1}$ denote the transpose and the inverse of $\mathbf{A}$, respectively. If $\mathbf{A}$ and $\mathbf{B}$ are symmetric matrices, $\mathbf{A}>\mathbf{B}$ means that $\mathbf{A}-\mathbf{B}$ is positive definite matrix. $\lambda_{\max }(\mathbf{A})$ and $\lambda_{\min }(\mathbf{A})$ denote the maximum eigenvalue and the minimum eigenvalue of matrix A, respectively. I denotes the identity matrix with appropriate dimensions. For any interval $J \subseteq \mathbb{R}$, set $S \subseteq \mathbb{R}^{k}(1 \leq k \leq n), \quad C(J, S)=\{\varphi: J \rightarrow S$ is continuous $\}$ and $C^{1}(J, S)=\{\varphi: J \rightarrow S$ is continuously differentiable $\} . \quad \Lambda=$ $\{1,2, \ldots, n\}$. Notation $\star$ always denotes the symmetric block in a symmetric matrix.

Consider the following neural networks with time delay

$$
\begin{aligned}
\dot{x}(t) & =-A x(t)+D f(x(t))+E g(x(t-\tau))+v(t), \quad t>0, \\
\mathbf{z}_{1}(t) & =\mathbf{C} x(t), \\
\mathbf{x}(t) & =\phi(t), \quad t \in[-\tau, 0],
\end{aligned}
$$

where $\mathbf{x}(t)=\left(x_{1}(t), x_{2}(t), \ldots, x_{n}(t)\right)^{T} \in \mathbb{R}^{n}$ is the state vector of the network networks; $n$ corresponds to the number of neurons; $v(t) \in \mathbb{R}^{n}$ is an external input; $\mathbf{z}_{1}(t) \in \mathbb{R}^{l}$ is the output vector; $\phi(t) \in C^{1}\left([-\tau, 0], \mathbb{R}^{n}\right)$ denotes a vector-valued initial function; $A=\operatorname{diag}\left(a_{1}, a_{2}, \ldots, a_{n}\right)>0$ is the self-feedback term; $D=\left(d_{i j}\right)_{n \times n}, E=\left(e_{i j}\right)_{n \times n}$, and $\mathbf{C} \in \mathbb{R}^{l \times n}$ represent the connection weight matrix; $\tau>0$ is the transmission constant delay; and $f=\left(f_{1}, \ldots, f_{n}\right)^{T}$ and $g=\left(g_{1}, \ldots, g_{n}\right)^{T}$ represent neuron activation functions satisfying

$$
\begin{aligned}
& \left|f_{j}\left(\alpha_{1}\right)-f_{j}\left(\alpha_{2}\right)\right| \leq l_{j}^{f}\left|\alpha_{1}-\alpha_{2}\right|, \\
& \left|g_{j}\left(\alpha_{1}\right)-g_{j}\left(\alpha_{2}\right)\right| \leq l_{j}^{g}\left|\alpha_{1}-\alpha_{2}\right|,
\end{aligned}
$$

for any $\alpha_{1} \neq \alpha_{2}, j \in \Lambda$, where $l_{j}^{f}$ and $l_{j}^{g}$ are some positive constants, and for all $j \in \Lambda, f_{j}(0)=g_{j}(0)=0$. Define $L^{f} \doteq$ $\operatorname{diag}\left(l_{1}^{f}, \ldots, l_{n}^{f}\right)$ and $L^{g} \dot{\doteq} \operatorname{diag}\left(l_{1}^{g}, \ldots, l_{n}^{g}\right)$.

In this paper, we consider neural networks (1) as the master system, and the corresponding slave system is given as follows:

$$
\begin{aligned}
\dot{y}(t) & =-A y(t)+D f(y(t))+E g(y(t-\tau))+v(t)+\mathbf{B} u(t), \quad t>0, \\
\mathbf{z}_{2}(t) & =C y(t), \\
\mathbf{y}(t) & =\varphi(t), \quad t \in[-\tau, 0],
\end{aligned}
$$

where $\mathbf{y}(t)=\left(y_{1}(t), y_{2}(t), \ldots, y_{n}(t)\right)^{T} \in \mathbb{R}^{n}$ is the state vector, $\mathbf{z}_{2}(t) \in \mathbb{R}^{l}$ is the output vector, $\varphi(t) \in C^{1}\left([-\tau, 0], \mathbb{R}^{n}\right)$ denotes a vector-valued initial function, $\mathbf{B} \in \mathbb{R}^{n \times m}$ is constant matrix, and $u(t) \in \mathbb{R}^{m}$ represents the control input that will be designed.

Defining $e(t)=y(t)-x(t)$ as the synchronization error of master system (1) and the slave system (3), we get the following error system of neural networks:

$$
\begin{aligned}
& \dot{e}(t)=-A e(t)+D \bar{f}(e(t))+E \bar{g}(e(t-\tau))+B u(t), \quad t>0, \\
& z(t)=C e(t), \\
& e(t)=\psi(t), \quad t \in[-\tau, 0],
\end{aligned}
$$

where $\bar{f}(e(t))=f(y(t))-f(x(t)), \bar{g}(e(t-\tau))=g(y(t-\tau))-$ $g(x(t-\tau)), z(t)=z_{2}(t)-z_{1}(t)$, and $\psi(t)=\varphi(t)-\phi(t)$ are the initial condition of system (4).

In order to achieve synchronization between system (1) and system (3), we design the following aperiodically intermittent dynamic output feedback controller with time delay:

$$
\begin{aligned}
& \dot{\bar{e}}(t)=A_{l} \overline{\mathbf{e}}(t)+H_{l} \overline{\mathbf{e}}(t-\sigma)+B_{l} z(t)+E_{l} z(t-\sigma), \\
& u(t)=\mathbf{G}_{l} \overline{\mathbf{e}}(t)+\mathbf{F}_{l} \overline{\mathbf{e}}(t-\sigma)+\mathbf{D}_{l} z(t),
\end{aligned}
$$

and $A_{l}, H_{l}, B_{l}, E_{l}, G_{l}, F_{l}, D_{l}$ satisfy

$$
\mathscr{H}_{l}=\left\{\begin{array}{l}
\mathscr{H}_{l}^{1}, t \in\left[t_{k}, t_{k}+\delta_{k}\right], \quad k \in \mathbb{Z}_{+}, \\
\mathscr{H}_{l}^{2}, t \in\left(t_{k}+\delta_{k}, t_{k+1}\right)
\end{array}\right.
$$

where $\overline{\mathbf{e}}(t) \in \mathbb{R}^{n}$ is the state vector of the controller (5), $\mathscr{H}_{l} \in\left\{A_{l}, H_{l}, B_{l}, E_{l}, G_{l}, F_{l}, D_{l}\right\}, A_{l}^{i}, H_{l}^{i}, B_{l}^{i}, E_{l}^{i}(i=1,2)$, and $\mathbf{G}_{l}^{1}, \mathbf{F}_{l}^{1}, \mathbf{D}_{l}^{1} \in \mathbb{R}^{n}$ are unknown control gain matrices, $\mathbf{G}_{l}^{2}=$ $\mathbf{F}_{l}^{2}=\mathbf{D}_{l}^{2}=0, \sigma>0$ is the constant delay.

Define $\xi(t)=\left[e^{T}(t), \bar{e}^{T}(t)\right]^{T}$, combining (4) and (5), one can obtain the following closed-loop system:

$$
\dot{\xi}(t)= \begin{cases}A_{1} \xi(t)+B_{1} \xi(t-\sigma)+\bar{D} \bar{f}(\xi(t))+\bar{E} \bar{g}(\xi(t-\tau)), & t \in\left[t_{k}, t_{k}+\delta_{k}\right], \\ A_{2} \xi(t)+B_{2} \xi(t-\sigma)+\bar{D} \bar{f}(\xi(t))+\bar{E} \bar{g}(\xi(t-\tau)), & t \in\left(t_{k}+\delta_{k}, t_{k+1}\right),\end{cases}
$$


where

$$
\begin{aligned}
& A_{1}=\left(\begin{array}{cc}
-A+B D_{l}^{1} C & B G_{l}^{1} \\
B_{l}^{1} C & A_{l}^{1}
\end{array}\right) \text {, } \\
& \bar{g}(\xi(t-\tau))=\left(\begin{array}{c}
\bar{g}(e(t-\tau)) \\
0
\end{array}\right), \\
& A_{2}=\left(\begin{array}{cc}
-A & 0 \\
B_{l}^{2} C & A_{l}^{2}
\end{array}\right) \text {, } \\
& B_{1}=\left(\begin{array}{cc}
0 & B F_{l}^{1} \\
E_{l}^{1} C & H_{l}^{1}
\end{array}\right) \text {, } \\
& \bar{D}=\left(\begin{array}{ll}
D & 0 \\
0 & 0
\end{array}\right) \text {, } \\
& B_{2}=\left(\begin{array}{cc}
0 & 0 \\
E_{l}^{2} C & H_{l}^{2}
\end{array}\right) \text {, } \\
& \bar{E}=\left(\begin{array}{ll}
E & 0 \\
0 & 0
\end{array}\right) \text {, } \\
& \bar{f}(\xi(t))=\left(\begin{array}{c}
\bar{f}(e(t)) \\
0
\end{array}\right) .
\end{aligned}
$$

To derive the main results, we will introduce the following definition and lemmas.

Definition 1 [17]. The master system (1) and the slave system (3) are said to be exponential synchronization, if there exist constant scalars $\lambda>0$ and $N>0$ such that every solution $\xi(t)$ of the system (7) satisfies

$$
|\xi(t)| \leq N\|\psi\|_{\tau} e^{-\lambda t}
$$

where the constant $\lambda$ is defined as the exponential synchronization rate and $\|\psi\|_{\tau}=\sup _{-\tau \leq s \leq 0}\{|\xi(s)|,|\dot{\xi}(s)|\}$.

Lemma 1 [45]. For any $n \times n$ matrix $\mathbf{R}>0$, scalar $h>0$ and a vector function $\boldsymbol{\sigma}(\cdot):[-h, 0] \rightarrow \mathbb{R}^{n}$, such that the integrations concerned are well defined, then the following inequality is hold:

$$
h \int_{-h}^{0} \boldsymbol{\sigma}^{T}(s) \mathbf{R} \boldsymbol{\sigma}(s) d s \geq \int_{-h}^{0} \boldsymbol{\sigma}^{T}(s) d s \mathbf{R} \int_{-h}^{0} \boldsymbol{\sigma}(s) d s .
$$

Lemma 2 [10]. Given matrices $\mathbf{A}, \mathbf{B}$, and $\mathbf{C}$ with $\mathbf{A}^{T}=\mathbf{A}$ and $\mathbf{C}^{T}=\mathbf{C}$, then

$$
\left[\begin{array}{ll}
\mathbf{A} & \mathbf{B} \\
\star & \mathbf{C}
\end{array}\right]<0,
$$

is equivalent to one of the following conditions:

(1) $\mathbf{A}<0$ and $\mathbf{C}-\mathbf{B}^{T} \mathbf{A}^{-1} \mathbf{B}<0$.

(2) $\mathbf{C}<0$ and $\mathbf{A}-\mathbf{B C}^{-1} \mathbf{B}^{T}<0$.

\section{Main Results}

3.1. Exponential Synchronization of Master-Slave Systems. In this section, we shall investigate the exponential synchronization of systems (1) and (3) by constructing suitable Lyapunov-Krasovskii functionals and applying the piecewise analytic method and LMI technique. For the convenience of presentation, in the following, we denote $T_{k}=t_{k+1}-t_{k}, \eta_{k}=\delta_{k} / T_{k}$, and $\bar{\eta}=\inf _{k \in \mathbb{Z}_{+}} \eta_{k}, k \in \mathbb{Z}_{+}$, where $0<\eta_{k}<1$ is called the control rate of the $k$ th control period.

Theorem 1. The master system (1) and the slave system (3) achieve exponential synchronization under aperiodically intermittent control dynamic output feedback controller (5), if for given scalars $\alpha>0$ and $\beta>0$, there exist $2 n \times$ $2 n$ symmetric positive definite matrices $\mathbf{P}>0, \mathbf{Q}_{1}>0, \mathbf{Q}_{2}>0$, $\mathbf{R}_{1}>0, \mathbf{R}_{2}>0, \mathbf{S}>0$, and $\mathbf{W}>0$ such that the following inequalities are hold:

$$
\mathscr{M}_{1}=\left(\begin{array}{ccc}
\Sigma & \tau \Gamma_{1}^{T} & \sigma \Gamma_{1}^{T} \\
\star & -R_{1}^{-1} & 0 \\
\star & \star & -R_{2}^{-1}
\end{array}\right)<0,
$$

$$
\mathscr{M}_{2}=\left(\begin{array}{ccc}
\Upsilon & \tau \Gamma_{2}^{T} & \sigma \Gamma_{2}^{T} \\
\star & -R_{1}^{-1} & 0 \\
\star & \star & -R_{2}^{-1}
\end{array}\right)<0
$$

$$
\lambda=\alpha \bar{\eta}-\beta(1-\bar{\eta})>0
$$

where

$$
\Sigma=\left(\begin{array}{ccccc}
\Sigma_{11} & e^{-2 \alpha \tau} R_{1} & \Sigma_{13} & P \bar{D} & P \bar{E} \\
\star & \Sigma_{22} & 0 & 0 & 0 \\
\star & \star & \Sigma_{33} & 0 & 0 \\
\star & \star & \star & -S & 0 \\
\star & \star & \star & \star & -W
\end{array}\right) \text {, }
$$




$$
\begin{aligned}
& \Upsilon=\left(\begin{array}{ccccc}
\Upsilon_{11} & e^{-2 \alpha \tau} R_{1} & \Upsilon_{13} & P \bar{D} & P \bar{E} \\
\star & \Sigma_{22} & 0 & 0 & 0 \\
\star & \star & \Sigma_{33} & 0 & 0 \\
\star & \star & \star & -S & 0 \\
\star & \star & \star & \star & -W
\end{array}\right), \\
& \Sigma_{11}=P A_{1}+A_{1}^{T} P+2 \alpha P+Q_{1}+Q_{2}-e^{-2 \alpha \tau} R_{1}-e^{-2 \alpha \sigma} R_{2}+\bar{L}^{\bar{f}}, \\
& \Sigma_{13}=P B_{1}+e^{-2 \alpha \sigma} R_{2}, \\
& \Sigma_{22}=-e^{-2 \alpha \tau} Q_{1}-e^{-2 \alpha \tau} R_{1}+\bar{L}^{\bar{g}}, \\
& \Sigma_{33}=-e^{-2 \alpha \sigma}\left(Q_{2}+R_{2}\right), \\
& \Upsilon_{11}=P A_{2}+A_{2}^{T} P-2 \beta P+Q_{1}+Q_{2}-e^{-2 \alpha \tau} R_{1}-e^{-2 \alpha \sigma} R_{2}+\bar{L}^{\bar{f}}, \\
& \Upsilon_{13}=P B_{2}+e^{-2 \alpha \sigma} R_{2}
\end{aligned}
$$

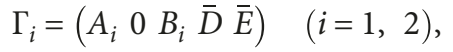

$$
\begin{aligned}
& S=\left(\begin{array}{cc}
S_{11} & S_{12} \\
\star & S_{22}
\end{array}\right) \\
& W=\left(\begin{array}{cc}
W_{11} & W_{12} \\
\star & W_{22}
\end{array}\right) .
\end{aligned}
$$

Proof 1. We consider the following Lyapunov-Krasovskii functional candidate for the error system (7) as

$$
\begin{aligned}
V\left(t, \xi_{t}\right)= & \xi^{T}(t) P \xi(t)+\int_{t-\tau}^{t} e^{2 \alpha(s-t)} \xi^{T}(s) Q_{1} \xi(s) d s \\
& +\int_{t-\sigma}^{t} e^{2 \alpha(s-t)} \xi^{T}(s) Q_{2} \xi(s) d s \\
& +\tau \int_{-\tau}^{0} \int_{t+\theta}^{t} e^{2 \alpha(s-t)} \dot{\xi}^{T}(s) R_{1} \dot{\xi}(s) d s d \theta \\
& +\sigma \int_{-\sigma}^{0} \int_{t+\theta}^{t} e^{2 \alpha(s-t)} \dot{\xi}^{T}(s) R_{2} \dot{\xi}(s) d s d \theta .
\end{aligned}
$$

It is easy to deduced that

$$
\lambda_{1}|\xi(t)|^{2} \leq V\left(t, \xi_{t}\right) \leq \lambda_{2}\left\|\xi_{t}\right\|_{\tau}^{2},
$$

where $\left\|\xi_{t}\right\|_{\tau}=\sup _{s \in[-\tau, 0]}\{|\xi(t+s)|,|\dot{\xi}(t+s)|\}$ and

$$
\begin{aligned}
\lambda_{1}= & \lambda_{\min }(P), \\
\lambda_{2}= & \lambda_{\max }(P)+\tau \lambda_{\max }\left(Q_{1}\right)+\sigma \lambda_{\max }\left(Q_{2}\right) \\
& +\tau^{2} \lambda_{\max }\left(R_{1}\right)+\sigma^{2} \lambda_{\max }\left(R_{2}\right) .
\end{aligned}
$$

Calculating the derivative of $V\left(t, \xi_{t}\right)$ with respect to $t$ along the trajectory of error system (7), it can be deduced that

$$
\begin{aligned}
\dot{V}\left(t, \xi_{t}\right)= & 2 \xi^{T}(t) P \dot{\xi}(t)+\xi^{T}(t)\left(Q_{1}+Q_{2}\right) \xi(t) \\
& -e^{-2 \alpha \tau} \xi^{T}(t-\tau) Q_{1} \xi(t-\tau) \\
& -e^{-2 \alpha \sigma} \xi^{T}(t-\sigma) Q_{2} \xi(t-\sigma) \\
& +\dot{\xi}^{T}(t)\left(\tau^{2} R_{1}+\sigma^{2} R_{2}\right) \dot{\xi}(t) \\
& -\tau \int_{t-\tau}^{t} e^{2 \alpha(s-t)} \dot{\xi}^{T}(s) R_{1} \dot{\xi}(s) d s \\
& -\sigma \int_{t-\sigma}^{t} e^{2 \alpha(s-t)} \dot{\xi}^{T}(s) R_{2} \dot{\xi}(s) d s \\
& -2 \alpha \int_{t-\tau}^{t} e^{2 \alpha(s-t)} \xi^{T}(s) Q_{1} \xi(s) d s \\
& -2 \alpha \int_{t-\sigma}^{t} e^{2 \alpha(s-t)} \xi^{T}(s) Q_{2} \xi(s) d s \\
& -2 \alpha \tau \int_{-\tau}^{0} \int_{t+\theta}^{t} e^{2 \alpha(s-t)} \dot{\xi}^{T}(s) R_{1} \dot{\xi}(s) d s d \theta \\
& -2 \alpha \sigma \int_{-\sigma}^{0} \int_{t+\theta}^{t} e^{2 \alpha(s-t)} \dot{\xi}^{T}(s) R_{2} \dot{\xi}(s) d s d \theta .
\end{aligned}
$$

Applying Lemma 1 and the Newton-Leibniz formula

$$
\int_{t-\tau}^{t} \dot{\xi}(s) d s=\xi(t)-\xi(t-\tau)
$$

we have

$$
\begin{aligned}
-\tau \int_{t-\tau}^{t} & e^{2 \alpha(s-t)} \dot{\xi}^{T}(s) R_{1} \dot{\xi}(s) d s \\
& \leq-\tau e^{-2 \alpha \tau} \int_{t-\tau}^{t} \dot{\xi}^{T}(s) R_{1} \dot{\xi}(s) d s \\
& \leq-e^{-2 \alpha \tau}\left(\int_{t-\tau}^{t} \dot{\xi}(s) d s\right)^{T} R_{1}\left(\int_{t-\tau}^{t} \dot{\xi}(s) d s\right) \\
& \leq-e^{-2 \alpha \tau}(\xi(t)-\xi(t-\tau))^{T} R_{1}(\xi(t)-\xi(t-\tau)) .
\end{aligned}
$$

Similarly, it holds that

$$
\begin{aligned}
& -\sigma \int_{t-\sigma}^{t} e^{2 \alpha(s-t)} \dot{\xi}^{T}(s) R \dot{\xi}(s) d s \\
& \quad \leq-e^{-2 \alpha \sigma}(\xi(t)-\xi(t-\sigma))^{T} R_{2}(\xi(t)-\xi(t-\sigma)) .
\end{aligned}
$$

It follows from (2) that

$$
\begin{aligned}
\bar{f}^{T}(\xi(t)) S \bar{f}(\xi(t)) & =\bar{f}^{T}(e(t)) S_{11} \bar{f}(e(t)) \leq e^{T}(t) L^{f} S_{11} L^{f} e(t) \\
& =\xi^{T}(t) \bar{L}^{\bar{f}} \xi(t),
\end{aligned}
$$


and

$$
\begin{aligned}
\bar{g}^{T}(\xi(t-\tau)) W \bar{g}(\xi(t-\tau)) & =\bar{g}^{T}(e(t-\tau)) W_{11} \bar{g}(e(t-\tau)) \\
& \leq e^{T}(t-\tau) L^{g} W_{11} L^{g} e(t-\tau) \\
& =\xi^{T}(t-\tau) \bar{L}^{\bar{g}} \xi(t-\tau)
\end{aligned}
$$

that is,

$$
\begin{aligned}
& 0 \leq \xi^{T}(t) \bar{L}^{\bar{f}} \xi(t)-\bar{f}^{T}(\xi(t)) S \bar{f}(\xi(t)), \\
& 0 \leq \xi^{T}(t-\tau) \bar{L}^{\bar{g}} \xi(t-\tau)-\bar{g}^{T}(\xi(t-\tau)) W \bar{g}(\xi(t-\tau)),
\end{aligned}
$$

where

$$
\begin{aligned}
\bar{L}^{\bar{f}} & =\left(\begin{array}{cc}
L^{f} S_{11} L^{f} & 0 \\
0 & 0
\end{array}\right), \\
\bar{L}^{\bar{g}} & =\left(\begin{array}{cc}
L^{g} W_{11} L^{g} & 0 \\
0 & 0
\end{array}\right) .
\end{aligned}
$$

Furthermore, it follows from the trajectory equation of error system (7) that

$$
\dot{\xi}^{T}(t) \bar{R} \dot{\xi}(t)= \begin{cases}\omega^{T}(t) \Omega_{1} \omega(t), & t \in\left[t_{k}, t_{k}+\delta_{k}\right], \\ \omega^{T}(t) \Omega_{2} \omega(t), & t \in\left(t_{k}+\delta_{k}, t_{k+1}\right),\end{cases}
$$

where $\omega(t)=[\xi(t), \xi(t-\tau), \xi(t-\sigma), \bar{f}(\xi(t)), \bar{g}(\xi(t-\tau))]$, $\bar{R}=\tau^{2} R_{1}+\sigma^{2} R_{2}, \Omega_{1}=\Gamma_{1}^{T} \bar{R} \Gamma_{1}$, and $\Omega_{2}=\Gamma_{2}^{T} \bar{R} \Gamma_{2}$.

When $t \in\left[t_{k}, t_{k}+\delta_{k}\right], k \in \mathbb{Z}_{+}$, the slave system runs in control windows, thus the dynamic output feedback control works. Substituting (21), (22), and (25) into (19) and taking (27) into account, we obtain that

$$
\begin{aligned}
\dot{V}\left(t, \xi_{t}\right)+ & 2 \alpha V\left(t, \xi_{t}\right) \\
\leq & \xi^{T}(t)\left(P A_{1}+A_{1}^{T} P+2 \alpha P+Q_{1}+Q_{2}-e^{-2 \alpha \tau} R_{1}\right. \\
& \left.-e^{-2 \alpha \sigma} R_{2}+\bar{L}^{\bar{f}}\right) \xi(t)+2 e^{-2 \alpha \tau} \xi^{T}(t) R_{1} \xi(t-\tau) \\
& +2 \xi^{T}(t)\left(P B_{1}+e^{-2 \alpha \sigma} R_{2}\right) \xi(t-\sigma) \\
& +2 \xi^{T}(t) P \bar{D} \bar{f}(\xi(t))+2 \xi^{T}(t) P \bar{E} \bar{g}(\xi(t-\tau)) \\
& +\xi^{T}(t-\tau)\left(-e^{-2 \alpha \tau} Q_{1}-e^{-2 \alpha \tau} R_{1}+\bar{L}^{\bar{g}}\right) \xi(t-\tau) \\
& -e^{-2 \alpha \sigma} \xi^{T}(t-\sigma)\left(Q_{2}+R_{2}\right) \xi(t-\sigma) \\
& +\dot{\xi}^{T}(t)\left(\tau^{2} R_{1}+\sigma^{2} R_{2}\right) \dot{\xi}(t)-\bar{f}^{T}(\xi(t)) S \bar{f}(\xi(t)) \\
& -\bar{g}^{T}(\xi(t-\tau)) W \bar{g}(\xi(t-\tau)) \\
= & \omega^{T}(t)\left(\Sigma+\Omega_{1}\right) \omega(t) .
\end{aligned}
$$

If the matrix inequality (12) is satisfied, by Lemma 2 , it is equivalent to $\Sigma+\Omega_{1}<0$, then we get

$$
\dot{V}\left(t, \xi_{t}\right)<-2 \alpha V\left(t, \xi_{t}\right)
$$

Integrating both sides of the inequality (29) with respect to $t$ over the time interval $\left[t_{k}, t_{k}+\delta_{k}\right], k \in \mathbb{Z}_{+}$, we have

$$
V\left(t, \xi_{t}\right) \leq V\left(\xi\left(t_{k}\right)\right) e^{-2 \alpha\left(t-t_{k}\right)}
$$

In addition, when $\left(t_{k}+\delta_{k}, t_{k+1}\right), k \in \mathbb{Z}_{+}$, the slave system runs in free windows and the dynamic output feedback control does not work. We can get similarly,

$$
\begin{aligned}
\dot{V}\left(t, \xi_{t}\right)+ & \alpha \alpha\left(t, \xi_{t}\right) \\
\leq & \xi^{T}(t)\left(P A_{2}+A_{2}^{T} P-2 \beta P+Q_{1}+Q_{2}-e^{-2 \alpha \tau} R_{1}\right. \\
& \left.-e^{-2 \alpha \sigma} R_{2}+\bar{L}^{\bar{f}}\right) \xi(t)+2 e^{-2 \alpha \tau} \xi^{T}(t) R_{1} \xi(t-\tau) \\
& +2 \xi^{T}(t)\left(P B_{2}+e^{-2 \alpha \sigma} R_{2}\right) \xi(t-\sigma) \\
& +2 \xi^{T}(t) P \bar{D} \bar{f}(\xi(t))+2 \xi^{T}(t) P \bar{E} \bar{g}(\xi(t-\tau)) \\
& +\xi^{T}(t-\tau)\left(-e^{-2 \alpha \tau} Q_{1}-e^{-2 \alpha \tau} R_{1}+\tilde{L}^{\bar{g}}\right) \\
& \times \xi(t-\tau)-e^{-2 \alpha \sigma} \xi^{T}(t-\sigma)\left(Q_{2}+R_{2}\right) \xi(t-\sigma) \\
& +\dot{\xi}(t)\left(\tau^{2} R_{1}+\sigma^{2} R_{2}\right) \dot{\xi}(t)-\bar{f}^{T}(\xi(t)) S \bar{f}(\xi(t)) \\
& -\bar{g}^{T}(\xi(t-\tau)) W \bar{g}(\xi(t-\tau)) \\
& +(2 \alpha+2 \beta) \xi^{T}(t) P \xi(t) \\
\leq & \omega^{T}(t)\left(\Upsilon+\Omega_{2}\right) \omega(t)+(2 \alpha+2 \beta) \xi^{T}(t) P \xi(t) \\
\leq & \omega^{T}(t)\left(\Upsilon+\Omega_{2}\right) \omega(t)+(2 \alpha+2 \beta) V\left(t, \xi_{t}\right) .
\end{aligned}
$$

If the matrix inequality (13) is satisfied, by Lemma 2 , it is equivalent to $\Upsilon+\Omega_{2}<0$, then we get

$$
\dot{V}\left(t, \xi_{t}\right)<2 \beta V\left(t, \xi_{t}\right),
$$

therefore, when $t \in\left(t_{k}+\delta_{k}, t_{k+1}\right), k \in \mathbb{Z}_{+}$,

$$
V\left(t, \xi_{t}\right) \leq V\left(\xi\left(t_{k}+\delta_{k}\right)\right) e^{2 \beta\left(t-t_{k}-\delta_{k}\right)} .
$$

Combining with (29) and (32), by applying mathematical induction and the piecewise analytic method, we can obtain

$$
\begin{aligned}
V\left(\xi\left(t_{k+1}\right)\right) & \leq V\left(\xi\left(t_{k}+\delta_{k}\right)\right) e^{2 \beta\left(t_{k+1}-t_{k}-\delta_{k}\right)} \\
& \leq V\left(\xi\left(t_{k}\right)\right) e^{-2 \alpha \delta_{k}} e^{2 \beta\left(t_{k+1}-t_{k}-\delta_{k}\right)} \\
& =V\left(\xi\left(t_{k}\right)\right) e^{-2\left[\alpha \eta_{k}-\beta\left(1-\eta_{k}\right)\right] T_{k}} \ldots \\
& \leq V(\xi(0)) e^{\sum_{j=1}^{k}-2\left[\alpha \eta_{j}-\beta\left(1-\eta_{j}\right)\right] T_{j}}
\end{aligned}
$$

Thus, for $t \in\left[t_{k}, t_{k}+\delta_{k}\right], k \in \mathbb{Z}_{+}$, we have

$$
\begin{aligned}
V\left(t, \xi_{t}\right) & \leq V\left(\xi\left(t_{k}\right)\right) e^{-2 \alpha\left(t-t_{k}\right)} \\
& \leq V(\xi(0)) e^{\sum_{j=1}^{k-1}-2\left[\alpha \eta_{j}-\beta\left(1-\eta_{j}\right)\right] T_{j}} e^{-2 \alpha\left(t-t_{k}\right)} \\
& \leq V(\xi(0)) e^{-2[\alpha \bar{\eta}-\beta(1-\bar{\eta})] \sum_{j=1}^{k-1} T_{j}} e^{-2 \alpha\left(t-t_{k}\right)} \\
& =V(\xi(0)) e^{-2[\alpha \bar{\eta}-\beta(1-\bar{\eta})] t_{k}} e^{2 \alpha t_{k}} e^{-2 \alpha t} \\
& =V(\xi(0)) e^{2(\alpha+\beta)(1-\bar{\eta}) t_{k}} e^{-2 \alpha t} \leq V(\xi(0)) e^{-2[\alpha \bar{\eta}-\beta(1-\bar{\eta})] t} \\
& =V(\xi(0)) e^{-2 \lambda t},
\end{aligned}
$$


and for $t \in\left(t_{k}+\delta_{k}, t_{k+1}\right), k \in \mathbb{Z}_{+}$,

$$
\begin{aligned}
V\left(t, \xi_{t}\right) & \leq V\left(\xi\left(t_{k}+\delta_{k}\right)\right) e^{2 \beta\left(t-t_{k}-\delta_{k}\right)} \leq V\left(\xi\left(t_{k}\right)\right) e^{-2 \alpha \delta_{k}} e^{2 \beta\left(t-t_{k}-\delta_{k}\right)} \\
& \leq V\left(\xi\left(t_{k}\right)\right) e^{-2 \alpha \delta_{k}} e^{2 \beta\left(t_{k+1}-t_{k}-\delta_{k}\right)} \\
& \leq V(\xi(0)) e^{-2[\alpha \bar{\eta}-\beta(1-\bar{\eta})] \sum_{j=1}^{k} T_{j}}=V(\xi(0)) e^{-2[\alpha \bar{\eta}-\beta(1-\bar{\eta})] t_{k+1}} \\
& \leq V(\xi(0)) e^{-2[\alpha \bar{\eta}-\beta(1-\bar{\eta})] t}=V(\xi(0)) e^{-2 \lambda t} .
\end{aligned}
$$

From (35) and (36), we get

$$
V\left(t, \xi_{t}\right) \leq V(\xi(0)) e^{-2 \lambda t}
$$

Furthermore, it follows from (17) that

$$
\lambda_{1}|\xi(t)|^{2} \leq V\left(t, \xi_{t}\right) \leq V(\xi(0)) e^{-2 \lambda t} \leq \lambda_{2} e^{-2 \lambda t}\|\psi\|_{\tau}^{2}
$$

Hence, we finally obtain that

$$
|\xi(t)| \leq \sqrt{\frac{\lambda_{2}}{\lambda_{1}}}\|\psi\|_{\tau} e^{-\lambda t} .
$$

By Definition 1, the master system (1) can be globally exponentially synchronized with the slave system (3) under the controller (5). The proof is completed.

Remark 1. The LMI method is the most common method to investigate the dynamic behavior of delayed neural networks owing to its large number of advantages, such as the criterion contains many unknown parameters and has great degree of freedom; it can be realized by the LMI toolbox in MATLAB software and can analyze the upper and lower bounds of the time delay; it can add constraints appropriately and is suitable for designing the controller in delayed neural networks and so on. The realization of LMI is that it is a convex optimization problem and is capable to provide the desired performance analysis [46-49]. It can be said that the LMI method is the mainstream in current research methods of dynamic behavior of delayed neural networks. Therefore, the criterion of Theorem 1 is effectively derived through LMI technique.

Remark 2. A more flexible selection strategy based on the control rate is presented. Under the proposed strategy, synchronization of neural networks is more effectively achieved in complex environment. In particular, it is easy to see that the control rates of different control periods are different. Furthermore, two free weight matrices, $\mathbf{S}$ and $\mathbf{W}$, are introduced in the derivation of Theorem 1, which is helpful to enhance the feasible solution of the derived synchronization criteria.

Remark 3. It is noted that the results of [35-39] are valid with periodically intermittent control. While in this paper, Theorem 1 is effective with aperiodically intermittent control, which is more general and flexible than that of [35-39]. On the other hand, in [29-34], the transmittal time is ignored when constructing the controller. While in this paper, we fully consider the time delay when constructing the controller, which demonstrates that the results in [29-34] cannot be applied to this paper. Therefore, the obtained results are more effective than those in the above listed references.

Furthermore, if we consider that the control period and control width of each work time are uniformly fixed, that is, $T_{k}=T, \delta_{k}=\delta$, and $\eta_{k}=\eta$, where $T>0, \delta>0$, and $0<\eta<1$ are constants, then the delayed aperiodically intermittent dynamic output feedback controller is transformed into the following delayed periodically intermittent control dynamic output feedback controller:

$$
\begin{aligned}
& \dot{\bar{e}}(t)=A_{l} \bar{e}(t)+H_{l} \bar{e}(t-\sigma)+B_{l} z(t)+E_{l} z(t-\sigma), \\
& u(t)=G_{l} \bar{e}(t)+F_{l} \bar{e}(t-\sigma)+D_{l} z(t),
\end{aligned}
$$

and $A_{l}, H_{l}, B_{l}, E_{l}, G_{l}, F_{l}, D_{l}$ satisfy

$$
\mathscr{H}_{l}=\left\{\begin{array}{l}
\mathscr{H}_{l}^{1}, t \in[m T, m T+\delta] \\
\mathscr{H}_{l}^{2}, t \in(m T+\delta,(m+1) T),
\end{array}\right.
$$

where $\mathscr{H}_{l} \in\left\{A_{l}, H_{l}, B_{l}, E_{l}, G_{l}, F_{l}, D_{l}\right\}, A_{l}^{i}, H_{l}^{i}, B_{l}^{i}, E_{l}^{i}(i=$ $1,2)$, and $\mathbf{G}_{l}^{1}, \mathbf{F}_{l}^{1}, \mathbf{D}_{l}^{1} \in \mathbb{R}^{n}$ are unknown control gain matrices, $\mathbf{G}_{l}^{2}=\mathbf{F}_{l}^{2}=\mathbf{D}_{l}^{2}=0, \quad m \in \mathbb{N}$. Based on Theorem 1 , then we can obtain the following corollary whose proof is omitted here.

Corollary 1. The master system (1) and the slave system (3) achieve exponential synchronization under the periodically intermittent control dynamic output feedback controller (40); if for given scalars $\alpha>0$ and $\beta>0$, there exist $2 n \times 2 n$ symmetric positive definite matrices $\mathbf{P}>0, \mathbf{Q}_{1}>0, \mathbf{Q}_{2}>0$, $\mathbf{R}_{1}>0, \mathbf{R}_{2}>0, \mathbf{S}>0$, and $\mathbf{W}>0$ such that (12), (13), and following inequality hold:

$$
\lambda=\alpha \eta-\beta(1-\eta)>0 .
$$

In particular, let $T_{k}=\delta_{k}, k \in \mathbb{Z}_{+}$, that is, $\eta_{k} \equiv 1$, then the delayed aperiodically intermittent dynamic output feedback controller is transformed into a more general delayed dynamic output feedback controller:

$$
\begin{aligned}
& \dot{\bar{e}}(t)=A_{l} \bar{e}(t)+H_{l} \bar{e}(t-\sigma)+B_{l} z(t)+E_{l} z(t-\sigma), \\
& u(t)=G_{l} \bar{e}(t)+F_{l} \bar{e}(t-\sigma)+D_{l} z(t),
\end{aligned}
$$

and $\mathbf{A}_{l}, \mathbf{H}_{l}, \mathbf{B}_{l}, \mathbf{E}_{l}, \mathbf{G}_{l}, \mathbf{F}_{l}, \mathbf{D}_{l}$ are unknown control gain matrices, which means that the control occurs in whole control period. Based on Theorem 1, then we can obtain the following corollary whose proof is omitted here.

Corollary 2. The master system (1) and the slave system (3) achieve exponential synchronization under the desired controller (43); if for given scalar $\alpha>0$, there exist $2 n \times 2 n$ symmetric positive definite matrices $\mathbf{P}>0, \mathbf{Q}_{1}>0, \mathbf{Q}_{2}>0$, $\mathbf{R}_{1}>0, \mathbf{R}_{2}>0, \mathbf{S}>0$, and $\mathbf{W}>0$ such that (12) holds.

3.2. Controller Design. Note that the controller gain matrices $\mathbf{A}_{l}^{1}, \mathbf{A}_{l}^{2}, \mathbf{H}_{l}^{1}, \mathbf{H}_{l}^{2}, \mathbf{B}_{l}^{1}, \mathbf{B}_{l}^{2}, \mathbf{E}_{l}^{1}, \mathbf{E}_{l}^{2}, \mathbf{G}_{l}^{1}, \mathbf{F}_{l}^{1}$, and $\mathbf{D}_{l}^{1}$ cannot be derived directly based on Theorem 1 due to the fact that 
they are coupled with the positive definite matrix $\mathbf{P}$ in (12) and (13). In order to calculate the controller gain matrices, we apply the partition matrix method to get the following conclusion.

Theorem 2. The controlled error system (7) is exponentially stable; if for given scalars $\varepsilon>0, \alpha>0$, and $\beta>0$, there exist $n \times n$ positive definite matrices $\mathbf{U}>0$ and $\mathbf{M}>0$, invertible matrices $\mathbf{H}$ and $\mathbf{Y}$, and $2 n \times 2 n$-real matrices $\tilde{\mathbf{Q}}_{1}>0, \tilde{\mathbf{Q}}_{2}>0$, $\tilde{\mathbf{R}}_{1}>0, \tilde{\mathbf{R}}_{2}>0, \mathbf{Z}>0$, and $\mathbf{G}_{j}(j=1,2,3,4,5,6,7,8,9,10,11)$ such that the (14) and the following conditions hold.

$$
\begin{aligned}
& \breve{\mathscr{M}}_{1}<0, \\
& \breve{\mathscr{M}}_{2}<0, \\
& Z=\left(\begin{array}{cc}
M & I \\
I & U
\end{array}\right)>0,
\end{aligned}
$$

where

$$
\begin{aligned}
& \breve{\mathscr{M}}_{1}=\left(\begin{array}{ccccccc}
\breve{\Sigma}_{11} & e^{-2 a \tau} \tilde{R}_{1} & \breve{\Sigma}_{13} & \Phi_{1} & \Phi_{2} & \tau \Phi_{0}^{T} & \sigma \Phi_{0}^{T} \\
\star & \breve{\Sigma}_{22} & 0 & 0 & 0 & 0 & 0 \\
\star & \star & \breve{\Sigma}_{33} & 0 & 0 & 0 & 0 \\
\star & \star & \star & -S & 0 & \tau \Phi_{1}^{T} & \sigma \Phi_{1}^{T} \\
\star & \star & \star & \star & -W & \tau \Phi_{2}^{T} & \sigma \Phi_{2}^{T} \\
\star & \star & \star & \star & \star & \breve{\Sigma}_{66} & 0 \\
\star & \star & \star & \star & \star & \star & \breve{\Sigma}_{77}
\end{array}\right), \\
& \breve{\mathscr{M}}_{2}=\left(\begin{array}{ccccccc}
\breve{\Upsilon}_{11} & e^{-2 a \tau} \tilde{R}_{1} & \breve{\Upsilon}_{13} & \Phi_{1} & \Phi_{2} & \tau \Phi_{0}^{T} & \sigma \Phi_{0}^{T} \\
\star & \breve{\Sigma}_{22} & 0 & 0 & 0 & 0 & 0 \\
\star & \star & \breve{\Sigma}_{33} & 0 & 0 & 0 & 0 \\
\star & \star & \star & -S & 0 & \tau \Phi_{1}^{T} & \sigma \Phi_{1}^{T} \\
\star & \star & \star & \star & -W & \tau \Phi_{2}^{T} & \sigma \Phi_{2}^{T} \\
\star & \star & \star & \star & \star & \breve{\Sigma}_{66} & 0 \\
\star & \star & \star & \star & \star & \star & \breve{\Sigma}_{77}
\end{array}\right),
\end{aligned}
$$

where

$$
\begin{aligned}
& \breve{\Sigma}_{11}=\Phi_{0}+\Phi_{0}^{T}+2 \alpha Z+\tilde{Q}_{1}+\tilde{Q}_{2}-e^{-2 \alpha \tau} \tilde{R}_{1}-e^{-2 \alpha \sigma} \tilde{R}_{2}+\breve{L}^{\breve{f}}, \\
& \breve{\Sigma}_{13}=\Phi_{3}+e^{-2 \alpha \sigma} \tilde{R}_{2}, \\
& \breve{\Sigma}_{22}=-e^{-2 \alpha \tau} \tilde{Q}_{1}-e^{-2 \alpha \tau} \tilde{R}_{1}+\breve{L}^{\breve{g}}, \\
& \breve{\Sigma}_{33}=-e^{-2 \alpha \sigma} \tilde{Q}_{2}-e^{-2 \alpha \sigma} \tilde{R}_{2},
\end{aligned}
$$

$\breve{\Sigma}_{66}=\varepsilon^{2} \tilde{R}_{1}-2 \varepsilon Z$,

$\breve{\Sigma}_{77}=\varepsilon^{2} \tilde{R}_{2}-2 \varepsilon Z$,

$\breve{\Upsilon}_{11}=\Phi_{4}+\Phi_{4}^{T}-2 \beta Z+\tilde{Q}_{1}+\tilde{Q}_{2}-e^{-2 \alpha \tau} \tilde{R}_{1}-e^{-2 \alpha \sigma} \tilde{R}_{2}+\breve{L}^{\breve{f}}$,

$\breve{\Upsilon}_{13}=\Phi_{5}+e^{-2 \alpha \sigma} \tilde{R}_{2}$,

$\Phi_{0}=\left(\begin{array}{cc}-A M+B G_{2} & -A+B G_{1} C \\ G_{4} & -U A+G_{3} C\end{array}\right)$,

$\Phi_{1}=\left(\begin{array}{cc}D & 0 \\ U D & 0\end{array}\right)$,

$\Phi_{2}=\left(\begin{array}{cc}E & 0 \\ U E & 0\end{array}\right)$,

$\Phi_{3}=\left(\begin{array}{cc}B G_{5} & 0 \\ G_{7} & G_{6} C\end{array}\right)$,

$\Phi_{4}=\left(\begin{array}{cc}-A M & -A \\ G_{9} & -U A+G_{8} C\end{array}\right)$,

$\Phi_{5}=\left(\begin{array}{cc}0 & 0 \\ G_{11} & G_{10} C\end{array}\right)$,

$\breve{L}^{\breve{f}}=\left(\begin{array}{cc}0 & 0 \\ 0 & L^{f} S_{11} L^{f}\end{array}\right)$,

$\breve{L}^{\breve{g}}=\left(\begin{array}{cc}0 & 0 \\ 0 & L^{g} W_{11} L^{g}\end{array}\right)$.

Moreover, the delayed aperiodically intermittent dynamic output feedback controller (5) is

$$
\begin{aligned}
D_{l}^{1} & =G_{1}, \\
G_{l}^{1} & =\left(G_{2}-G_{1} C M\right)\left(Y^{T}\right)^{-1}, \\
B_{l}^{1} & =H^{-1}\left(G_{3}-U B G_{1}\right), \\
A_{l}^{1} & =\left[G_{4}+U A M-G_{3} C M-U B\left(G_{2}-G_{1} C M\right)\right]\left(Y^{T}\right)^{-1}, \\
F_{l}^{1} & =G_{5}\left(Y^{T}\right)^{-1}, \\
E_{l}^{1} & =H^{-1} G_{6}, \\
H_{l}^{1} & =H^{-1}\left(G_{7}-G_{6} C M-U B G_{5}\right)\left(Y^{T}\right)^{-1}, \\
B_{l}^{2} & =H^{-1} G_{8}, \\
A_{l}^{2} & =H^{-1}\left(G_{9}+U A M-G_{8} C M\right)\left(Y^{T}\right)^{-1}, \\
E_{l}^{2} & =H^{-1} G_{10}, \\
H_{l}^{2} & =H^{-1}\left(G_{11}-G_{10} C M\right)\left(Y^{T}\right)^{-1} .
\end{aligned}
$$


Proof 2. Let $P$ and its inverse as

$$
\begin{gathered}
P=\left(\begin{array}{ll}
U & H \\
\star & V
\end{array}\right), \\
P^{-1}=\left(\begin{array}{cc}
M & Y \\
\star & W
\end{array}\right) .
\end{gathered}
$$

From $P P^{-1}=I$, we get

$$
U M+H Y^{T}=I, H^{T} M+V Y^{T}=0 .
$$

Set

$$
\begin{aligned}
& J_{1}=\left(\begin{array}{cc}
M & I \\
Y^{T} & 0
\end{array}\right), \\
& J_{2}=\left(\begin{array}{cc}
I & U \\
0 & H^{T}
\end{array}\right) .
\end{aligned}
$$

Then, we have

$$
\begin{gathered}
P J_{1}=J_{2}, \\
J_{1}^{T} P J_{1}=J_{1}^{T} J_{2} .
\end{gathered}
$$

Denote

$$
J=\operatorname{diag}\left\{J_{1}, J_{1}, J_{1}, I, I, J_{2}, J_{2}\right\},
$$

and

$$
\begin{aligned}
G_{1} & =D_{l}^{1}, \\
G_{2} & =G_{1} C M+G_{l}^{1} Y^{T}, \\
G_{3} & =U B G_{1}+H B_{l}^{1}, \\
G_{4} & =-U A M+G_{3} C M+U B\left(G_{2}-G_{1} C M\right)+H A_{l}^{1} Y^{T}, \\
G_{5} & =F_{l}^{1} Y^{T}, \\
G_{6} & =H E_{l}^{1}, \\
G_{7} & =G_{6} C M+U B G_{5}+H H_{l}^{1} Y^{T}, \\
G_{8} & =H B_{l}^{2}, \\
G_{9} & =-U A M+G_{8} C M+H A_{l}^{2} Y^{T}, \\
G_{10} & =H E_{l}^{2}, \\
G_{11} & =G_{10} C M+H H_{l}^{2} Y^{T} .
\end{aligned}
$$

Based on the concept of congruence transformation, the inequality (12) by premultiplying and postmultiplying the matrix $\mathbf{J}^{T}$ and $\mathbf{J}$, respectively, is equivalent to

$$
\tilde{\mathscr{M}}_{1}=\left(\begin{array}{ccccccc}
\tilde{\Sigma}_{11} & e^{-2 a \tau} \tilde{R}_{1} & \breve{\Sigma}_{13} & \Phi_{1} & \Phi_{2} & \tau \Phi_{0}^{T} & \sigma \Phi_{0}^{T} \\
\star & \tilde{\Sigma}_{22} & 0 & 0 & 0 & 0 & 0 \\
\star & \star & \breve{\Sigma}_{33} & 0 & 0 & 0 & 0 \\
\star & \star & \star & -S & 0 & \tau \Phi_{1}^{T} & \sigma \Phi_{1}^{T} \\
\star & \star & \star & \star & -W & \tau \Phi_{2}^{T} & \sigma \Phi_{2}^{T} \\
\star & \star & \star & \star & \star & \tilde{\Sigma}_{66} & 0 \\
\star & \star & \star & \star & \star & \star & \tilde{\Sigma}_{77}
\end{array}\right)
$$

where

$$
\begin{aligned}
& \tilde{\Sigma}_{11}=\Phi_{0}+\Phi_{0}^{T}+2 \alpha Z+\tilde{Q}_{1}+\tilde{Q}_{2}-e^{-2 \alpha \tau} \tilde{R}_{1}-e^{-2 \alpha \sigma} \tilde{R}_{2}+\tilde{L}^{\tilde{f}}, \\
& \tilde{\Sigma}_{22}=-e^{-2 \alpha \tau} \tilde{Q}_{1}-e^{-2 \alpha \tau} \tilde{R}_{1}+\tilde{L}^{\tilde{g}}, \\
& \tilde{\Sigma}_{66}=-Z \tilde{R}_{1}^{-1} Z, \\
& \tilde{\Sigma}_{77}=-Z \tilde{R}_{2}^{-1} Z, \\
& \tilde{Q}_{1}=J_{1}^{T} Q_{1} J_{1}, \\
& \tilde{Q}_{2}=J_{1}^{T} Q_{2} J_{1}, \\
& \tilde{R}_{1}=J_{1}^{T} R_{1} J_{1}, \\
& \tilde{R}_{2}=J_{1}^{T} R_{2} J_{1}, \\
& \tilde{L}^{\tilde{f}}=J_{1}^{T} \bar{L}^{\tilde{f}} J_{1}=\left(\begin{array}{cc}
M L^{f} S_{11} L^{f} M & M L^{f} S_{11} L^{f} \\
L^{f} S_{11} L^{f} M & L^{f} S_{11} L^{f}
\end{array}\right), \\
& \tilde{L}^{\tilde{g}}=J_{1}^{T} \bar{L}^{\bar{g}} J_{1}=\left(\begin{array}{cc}
M L^{g} W_{11} L^{g} M & M L^{g} W_{11} L^{g} \\
L^{g} W_{11} L^{g} M & L^{g} W_{11} L^{g}
\end{array}\right) .
\end{aligned}
$$

Similarly, the inequality (13) is equivalent to

$$
\tilde{\mathscr{M}}_{2}=\left(\begin{array}{ccccccc}
\tilde{\Upsilon}_{11} & e^{-2 a \tau} \tilde{R}_{1} & \breve{\Upsilon}_{13} & \Phi_{1} & \Phi_{2} & \tau \Phi_{0}^{T} & \sigma \Phi_{0}^{T} \\
\star & \tilde{\Sigma}_{22} & 0 & 0 & 0 & 0 & 0 \\
\star & \star & \breve{\Sigma}_{33} & 0 & 0 & 0 & 0 \\
\star & \star & \star & -S & 0 & \tau \Phi_{1}^{T} & \sigma \Phi_{1}^{T} \\
\star & \star & \star & \star & -W & \tau \Phi_{2}^{T} & \sigma \Phi_{2}^{T} \\
\star & \star & \star & \star & \star & \tilde{\Sigma}_{66} & 0 \\
\star & \star & \star & \star & \star & \star & \tilde{\Sigma}_{77}
\end{array}\right)
$$


where

$$
\tilde{\Upsilon}_{11}=\Phi_{4}+\Phi_{4}^{T}-2 \beta Z+\tilde{Q}_{1}+\tilde{Q}_{2}-e^{-2 \alpha \tau} \tilde{R}_{1}-e^{-2 \alpha \sigma} \tilde{R}_{2}+\tilde{L}^{\tilde{f}} .
$$

After elementary transformation, we have

$$
\begin{aligned}
&\left(\begin{array}{cc}
M L^{f} S_{11} L^{f} M & M L^{f} S_{11} L^{f} \\
L^{f} S_{11} L^{f} M & L^{f} S_{11} L^{f}
\end{array}\right) \Leftrightarrow\left(\begin{array}{cc}
0 & 0 \\
0 & L^{f} S_{11} L^{f}
\end{array}\right), \\
&\left(\begin{array}{cc}
M L^{g} W_{11} L^{g} M & M L^{g} W_{11} L^{g} \\
L^{g} W_{11} L^{g} M & L^{g} W_{11} L^{g}
\end{array}\right) \Leftrightarrow\left(\begin{array}{cc}
0 & 0 \\
0 & L^{g} W_{11} L^{g}
\end{array}\right) .
\end{aligned}
$$

For $\varepsilon>0, Z>0$, and $\tilde{R}^{-1}>0$, from $(\varepsilon \tilde{R}-Z) \tilde{R}^{-1}(\varepsilon \tilde{R}-Z)$ $\geq 0$, we can conclude that

$$
-Z \tilde{R}^{-1} Z \leq \varepsilon^{2} \tilde{R}-2 \varepsilon Z
$$

It results that $(56) \Leftrightarrow(44)$ and $(58) \Leftrightarrow(45)$. Therefore, all the conditions in Theorem 2 are satisfied. The proof is completed.

Remark 4. In Theorem 2, the delayed aperiodically intermittent dynamic output feedback control gain matrices are obtained by using the partition matrix method and elementary transformation of matrix. In particular, the partition matrix method provides a power tool to solve the problem of coupling between matrices. In addition, to linearize matrix inequalities, we use the matrix inequality (61), which guarantees the feasibility and effectiveness of the resulting criteria in terms of LMIs.

\section{Numerical Examples}

In this section, a numerical example and its numerical simulations are given to demonstrate the effectiveness and applicability of our exponential synchronization results.

Example 1. Consider the system (1) with $\tau=1, v(t)=[0 ; 0]$, $f(s)=g(s)=\tanh (s)$. The parameter matrices $\mathbf{A}, \mathbf{D}, \mathbf{E}, \mathbf{B}$, and $\mathbf{C}$ are given as follows:

$$
\begin{aligned}
& \mathbf{A}=\left(\begin{array}{ll}
1 & 0 \\
0 & 1
\end{array}\right), \\
& \mathbf{D}=\left(\begin{array}{cc}
2 & -0.1 \\
-5 & 0.75
\end{array}\right), \\
& \mathbf{E}=\left(\begin{array}{cc}
0.15 & 0.1 \\
0 & 0.3
\end{array}\right), \\
& \mathbf{B}=\left(\begin{array}{l}
0 \\
1
\end{array}\right), \\
& \mathbf{C}=\left(\begin{array}{ll}
1 & 1
\end{array}\right) .
\end{aligned}
$$

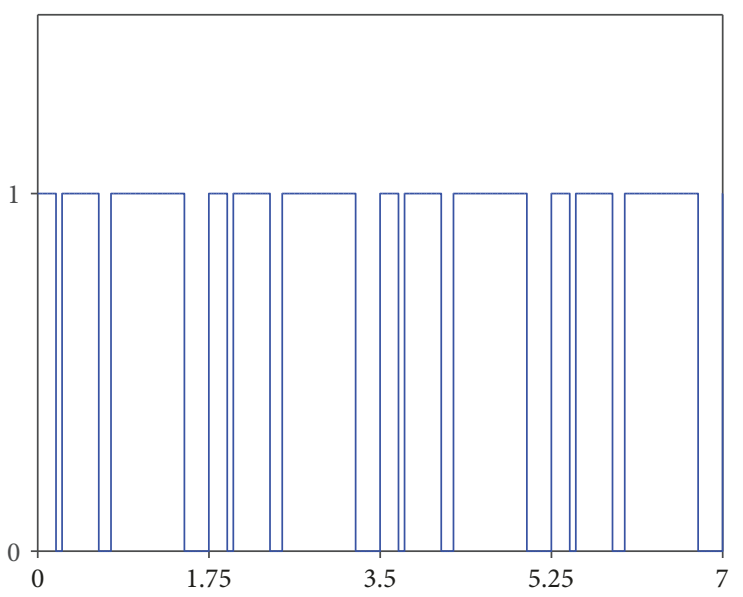

Figure 2: The intermittent control time sequence in Example 1.

Select the parameters as $\alpha=\beta=0.5, \varepsilon=10$, and $\sigma=0.5$. Figure 2 gives the intermittent control time sequence $t_{k}$, $k \in \mathbb{Z}_{+}$, satisfy

$$
\begin{aligned}
t_{7 k}-t_{7 k-1} & =0.25, \\
t_{7 k-1}-t_{7 k-2} & =0.75, \\
t_{7 k-2}-t_{7 k-3} & =0.125, \\
t_{7 k-3}-t_{7 k-4} & =0.375, \\
t_{7 k-4}-t_{7 k-5} & =0.0625, \\
t_{7 k-5}-t_{7 k-6} & =0.1875 .
\end{aligned}
$$

In this case, $\bar{\eta}=0.75$ and $L^{f}=L^{g}=\operatorname{diag}\{1,1\}$. It is easy to check that all conditions in Theorem 2 are true by using the LMI toolbox in MATLAB; then, the error system (7) is globally exponentially stable with the delayed aperiodically intermittent dynamic output feedback controller (5), that is, master system (1) can be exponentially synchronized with slave system (3), see Figure 3(a), and gain matrices in the desired controller (5) are derived as follows:

$$
\begin{aligned}
A_{l}^{1} & =\left(\begin{array}{cc}
-2.3640 & 13.8168 \\
0.5032 & -9.9442
\end{array}\right), \\
A_{l}^{2} & =\left(\begin{array}{cc}
-1.1632 & 0.9059 \\
1.0294 & -15.3119
\end{array}\right), \\
H_{l}^{1} & =\left(\begin{array}{ll}
-0.0761 & 1.0425 \\
-0.0203 & 0.2915
\end{array}\right), \\
H_{l}^{2} & =\left(\begin{array}{cc}
-0.0095 & 0.0702 \\
0.0022 & -0.0224
\end{array}\right), \\
B_{l}^{1} & =\left(\begin{array}{c}
69.2056 \\
-28.5133
\end{array}\right), \\
B_{l}^{2} & =\left(\begin{array}{c}
5.9345 \\
-54.3737
\end{array}\right),
\end{aligned}
$$




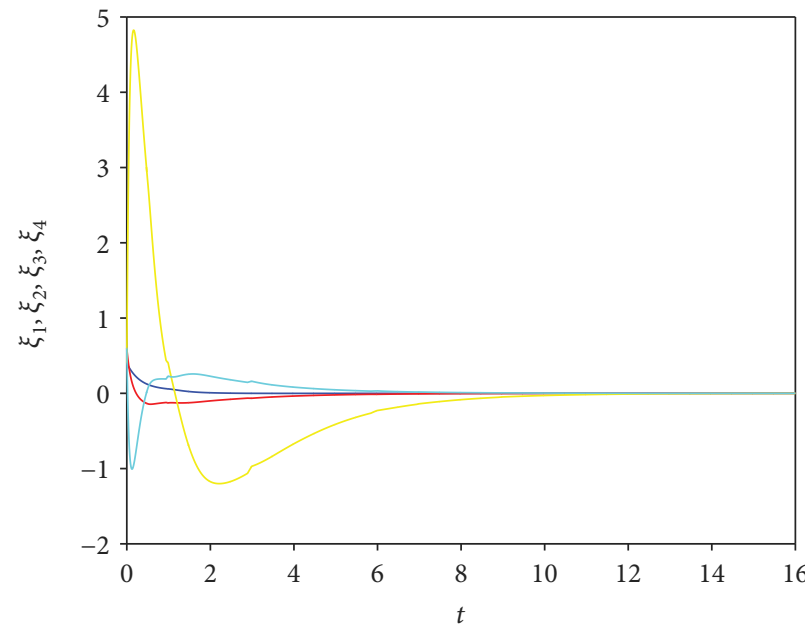

(a)

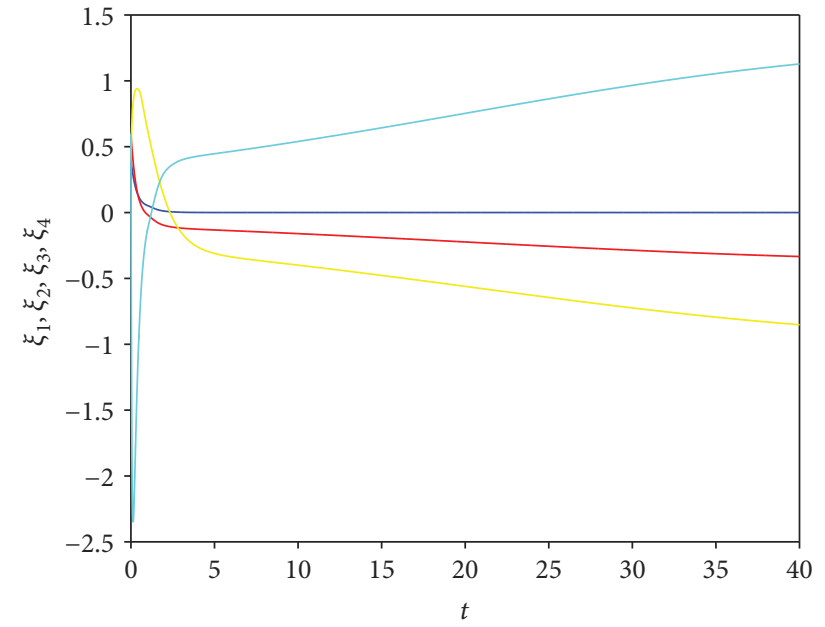

(b)

Figure 3: (a) State trajectories of error system (7) with controller (5). (b) State trajectories of error system (7) without controller (5).

$$
\begin{aligned}
& E_{l}^{1}=\left(\begin{array}{l}
0.4433 \\
0.0761
\end{array}\right), \\
& E_{l}^{2}=\left(\begin{array}{c}
0.3896 \\
-0.1311
\end{array}\right), \\
& G_{l}^{1}=\left(\begin{array}{ll}
0.0748-0.5794
\end{array}\right), \\
& D_{l}^{1}=-3.6757, \\
& F_{l}^{1}=\left(\begin{array}{ll}
0.0053-0.0817
\end{array}\right) .
\end{aligned}
$$

Under the same conditions, if there is no control input $u(t)$, in this case, Figure 3(b) tells us that the error system (7) is unstable and it shows the effectiveness of the desired controller (5).

\section{Conclusion}

In present paper, we have designed a new flexible feedback control method in complex environment: aperiodically intermittent dynamic output feedback control for exponential synchronization of neural networks by constructing suitable Lyapunov-Krasovskii functionals and applying the piecewise analytic method and LMI technique. In particular, we have fully considered the time delay when designing the controller, which can ensure that the obtained results have better application and less conservatism. Finally, an example is finally presented to show the effectiveness of obtained theoretical results. A possible application of the aperiodically intermittent dynamic output feedback control method in lag synchronization and finite-time synchronization of complex networks is expected to be discussed in the future.

\section{Conflicts of Interest}

The authors declare that they have no competing interests.

\section{Acknowledgments}

This work was supported by National Natural Science Foundation of China $(61673247,61773246)$ and the Research Fund for Distinguished Young Scholars and Excellent Young Scholars of Shandong Province (JQ201719, ZR2016JL024).

\section{References}

[1] L. O. Chua and L. Yang, "Cellular neural networks: applications," IEEE Transactions on Circuits and Systems, vol. 35, no. 10, pp. 1273-1290, 1988.

[2] S. Grossberg, "Nonlinear neural networks: principles, mechanisms, and architectures," Neural Networks, vol. 1, no. 1, pp. 17-61, 1988.

[3] Y.-H. Chen and S.-C. Fang, "Neurocomputing with time delay analysis for solving convex quadratic programming problems," IEEE Transactions on Neural Networks, vol. 11, no. 1, pp. 230-240, 2000.

[4] L. Zhang and Z. Yi, "Selectable and unselectable sets of neurons in recurrent neural networks with saturated piecewise linear transfer function," IEEE Transactions on Neural Networks, vol. 22, no. 7, pp. 1021-1031, 2011.

[5] X. Li and J. Cao, "An impulsive delay inequality involving unbounded time-varying delay and applications," IEEE Transactions on Automatic Control, vol. 62, no. 7, pp. 3618 3625, 2017.

[6] X. Li and J. Wu, "Stability of nonlinear differential systems with state-dependent delayed impulses," Automatica, vol. 64, pp. 63-69, 2016.

[7] O. M. Kwon, M. J. Park, J. H. Park, S. M. Lee, and E. J. Cha, "New and improved results on stability of static neural networks with interval time-varying delays," Applied Mathematics and Computation, vol. 239, pp. 346-357, 2014.

[8] O. M. Kwon, J. H. Park, S. M. Lee, and E. J. Cha, "New augmented Lyapunov-Krasovskii functional approach to stability analysis of neural networks with time-varying delays," Nonlinear Dynamics, vol. 76, no. 1, pp. 221-236, 2014.

[9] O.-M. Kwon, M.-J. Park, S.-M. Lee, J. H. Park, and E.-J. Cha, "Stability for neural networks with time-varying delays via 
some new approaches," IEEE Transactions on Neural Networks and Learning Systems, vol. 24, no. 2, pp. 181-193, 2013.

[10] S. Boyd, L. El Ghaoui, E. Feron, and V. Balakrishnan, Linear Matrix Inequalities in System and Control Theory, SIAM, Philadelphia, PA, USA, 1994.

[11] R. Rakkiyappan, S. Lakshmanan, R. Sivasamy, and C. P. Lim, "Leakage-delay-dependent stability analysis of Markovian jumping linear systems with time-varying delays and nonlinear perturbations," Applied Mathematical Modelling, vol. 40, no. 7-8, pp. 5026-5043, 2016.

[12] T. Huang, C. Li, S. Duan, and J. A. Starzyk, "Robust exponential stability of uncertain delayed neural networks with stochastic perturbation and impulse effects," IEEE Transactions on Neural Networks and Learning Systems, vol. 23, no. 6, pp. 866-875, 2012.

[13] R. Li and J. Cao, "Dissipativity analysis of memristive neural networks with time-varying delays and randomly occurring uncertainties," Mathematical Methods in the Applied Sciences, vol. 39, no. 11, pp. 2896-2915, 2016.

[14] I. Stamova, T. Stamov, and X. Li, "Global exponential stability of a class of impulsive cellular neural networks with supremums," International Journal of Adaptive Control and Signal Processing, vol. 28, no. 11, pp. 1227-1239, 2014.

[15] Y.-C. Wu, Q. Chaudhari, and E. Serpedin, "Clock synchronization of wireless sensor networks," IEEE Signal Processing Magazine, vol. 28, no. 1, pp. 124-138, 2011.

[16] R. Rakkiyappan, N. Sakthivel, and J. Cao, "Stochastic sampleddata control for synchronization of complex dynamical networks with control packet loss and additive time-varying delays," Neural Networks, vol. 66, pp. 46-63, 2015.

[17] R. Rakkiyappan, R. Sivasamy, and X. Li, "Synchronization of identical and nonidentical memristor-based chaotic systems via active backstepping control technique," Circuits, Systems, and Signal Processing, vol. 34, no. 3, pp. 763-778, 2015.

[18] X. Li, R. Rakkiyappan, and N. Sakthivel, "Non-fragile synchronization control for Markovian jumping complex dynamical networks with probabilistic time-varying coupling delays," Asian Journal of Control, vol. 17, no. 5, pp. 16781695, 2015.

[19] S. Chen and J. Cao, "Projective synchronization of neural networks with mixed time-varying delays and parameter mismatch," Nonlinear Dynamics, vol. 67, no. 2, pp. 13971406, 2012.

[20] T. Pyragienè and K. Pyragas, "Anticipating synchronization in a chain of chaotic oscillators with switching parameters," Physics Letters A, vol. 379, no. 47-48, pp. 3084-3088, 2015.

[21] L. Shi, H. Zhu, S. Zhong, K. Shi, and J. Cheng, "Function projective synchronization of complex networks with asymmetric coupling via adaptive and pinning feedback control," ISA Transactions, vol. 65, pp. 81-87, 2016.

[22] J. Lu, C. Ding, J. Lou, and J. Cao, "Outer synchronization of partially coupled dynamical networks via pinning impulsive controllers," Journal of the Franklin Institute, vol. 352, no. 11, pp. 5024-5041, 2015.

[23] D. Sadaoui, A. Boukabou, and S. Hadef, "Predictive feedback control and synchronization of hyperchaotic systems," Applied Mathematics and Computation, vol. 247, pp. 235-243, 2014.

[24] X. Zhang, X. Lv, and X. Li, "Sampled-data-based lag synchronization of chaotic delayed neural networks with impulsive control," Nonlinear Dynamics, vol. 90, no. 3, pp. 2199-2207, 2017.
[25] X. Li and S. Song, "Stabilization of delay systems: delaydependent impulsive control," IEEE Transactions on Automatic Control, vol. 62, no. 1, pp. 406-411, 2017.

[26] B. Liu, C. Dou, and D. J. Hill, "Robust exponential inputto-state stability of impulsive systems with an application in micro-grids," Systems \& Control Letters, vol. 65, pp. 64-73, 2014.

[27] X. Li and X. Fu, "Lag synchronization of chaotic delayed neural networks via impulsive control," IMA Journal of Mathematical Control and Information, vol. 29, no. 1, pp. 133-145, 2012.

[28] W. Xiong, R. Patel, J. Cao, and W. X. Zheng, "Synchronization of hierarchical time-varying neural networks based on asynchronous and intermittent sampled-data control," IEEE Transactions on Neural Networks and Learning Systems, vol. 28, no. 11, pp. 2837-2843, 2017.

[29] Y. Liu, C. Zhu, D. Chu, and W. Li, "Synchronization of stochastic coupled systems with time-varying coupling structure on networks via discrete-time state feedback control," Neurocomputing, vol. 285, pp. 104-116, 2018.

[30] A. T. Nguyen, P. Chevrel, and F. Claveau, "Gain-scheduled static output feedback control for saturated LPV systems with bounded parameter variations," Automatica, vol. 89, pp. 420424, 2018.

[31] Y. L. Wang and Q. L. Han, "Network-based modelling and dynamic output feedback control for unmanned marine vehicles in network environments," Automatica, vol. 91, pp. 43-53, 2018.

[32] Q. Qi and H. Zhang, "Output feedback control and stabilization for networked control systems with packet losses," IEEE Transactions on Cybernetics, vol. 47, no. 8, pp. 22232234, 2017.

[33] Y. Li, S. Tong, L. Liu, and G. Feng, “Adaptive output-feedback control design with prescribed performance for switched nonlinear systems," Automatica, vol. 80, pp. 225-231, 2017.

[34] H. K. Khalil, "Cascade high-gain observers in output feedback control," Automatica, vol. 80, pp. 110-118, 2017.

[35] N. Li and J. Cao, "Periodically intermittent control on robust exponential synchronization for switched interval coupled networks," Neurocomputing, vol. 131, pp. 52-58, 2014.

[36] Y. Fan, H. Liu, Y. Zhu, and J. Mei, "Fast synchronization of complex dynamical networks with time-varying delay via periodically intermittent control," Neurocomputing, vol. 205, pp. 182-194, 2016.

[37] X. H. Ma and J. A. Wang, "Pinning outer synchronization between two delayed complex networks with nonlinear coupling via adaptive periodically intermittent control," Neurocomputing, vol. 199, pp. 197-203, 2016.

[38] X. Liu, X. Shen, and H. Zhang, "Intermittent impulsive synchronization of chaotic delayed neural networks," Differential Equations and Dynamical Systems, vol. 19, no. 1-2, pp. 149-169, 2011.

[39] S. Zheng, "Intermittent impulsive projective synchronization in time-varying delayed dynamical network with variable structures," Complexity, vol. 21, no. S1, pp. 547-556, 2016.

[40] X. Liu and T. Chen, "Synchronization of complex networks via aperiodically intermittent pinning control," IEEE Transactions on Automatic Control, vol. 60, no. 12, pp. 3316-3321, 2015.

[41] X. Liu and T. Chen, "Synchronization of nonlinear coupled networks via aperiodically intermittent pinning control," IEEE Transactions on Neural Networks and Learning Systems, vol. 26, no. 1, pp. 113-126, 2015. 
[42] X. Liu and S. Li, "Cluster synchronization for linearly coupled nonidentical systems with delays via aperiodically intermittent pinning control," IEEE Access, vol. 5, pp. 4179-4189, 2017.

[43] Y. Wang and H. Yu, "Fuzzy synchronization of chaotic systems via intermittent control," Chaos, Solitons \& Fractals, vol. 106, pp. 154-160, 2018.

[44] Z.-M. Zhang, Y. He, M. Wu, and Q.-G. Wang, "Exponential synchronization of neural networks with time-varying delays via dynamic intermittent output feedback control," IEEE Transactions on Systems, Man, and Cybernetics: Systems, no. 99, pp. 1-11, 2017.

[45] K. Gu, "An integral inequality in the stability problem of timedelay systems," in Proceedings of the 39th IEEE Conference on Decision and Control (Cat. No. 00CH37187), pp. 2805-2018, Sydney, NSW, Australia, 2000.

[46] J. Cao and R. Li, "Fixed-time synchronization of delayed memristor-based recurrent neural networks," Science China Information Sciences, vol. 60, no. 3, article 032201, 2017.

[47] X. Li and S. Song, "Impulsive control for existence, uniqueness and global stability of periodic solutions of recurrent neural networks with discrete and continuously distributed delays," IEEE Transactions on Neural Networks and Learning Systems, vol. 24, no. 6, pp. 868-877, 2013.

[48] Q. Wang, Z. Wu, P. Shi, and A. Xue, "Robust control for switched systems subject to input saturation and parametric uncertainties," Journal of the Franklin Institute, vol. 354, no. 16, pp. 7266-7279, 2017.

[49] Y. Chen, S. Fei, and Y. Li, "Stabilization of neutral time-delay systems with actuator saturation via auxiliary time-delay feedback," Automatica, vol. 52, pp. 242-247, 2015. 


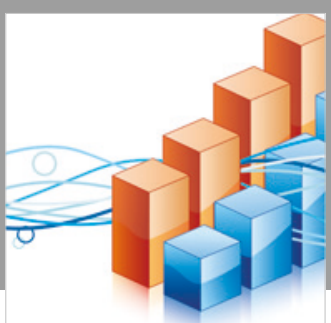

Advances in

Operations Research

\section{-n-m}
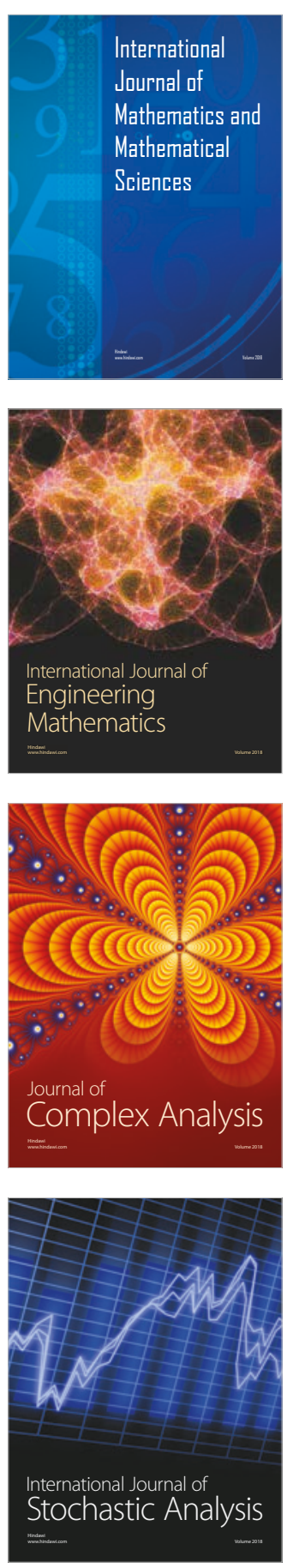
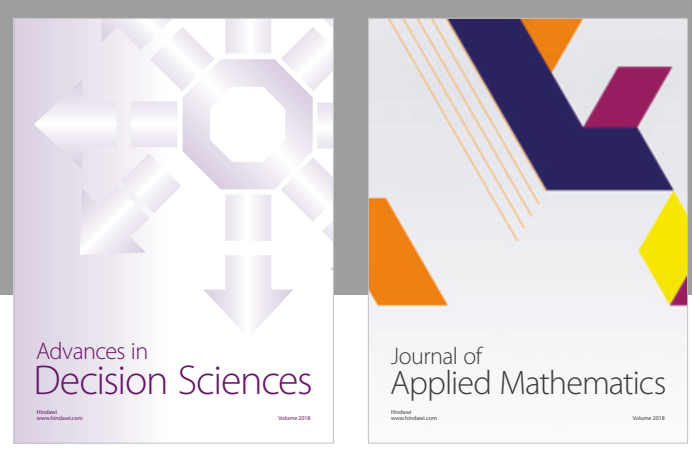

Journal of

Applied Mathematics
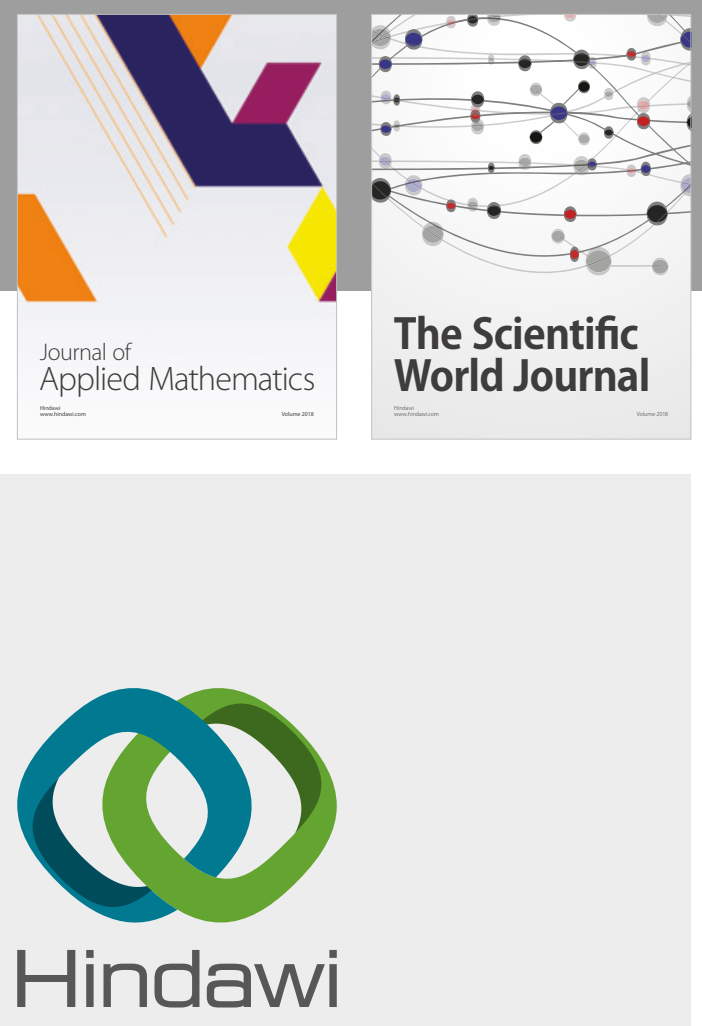

Submit your manuscripts at

www.hindawi.com

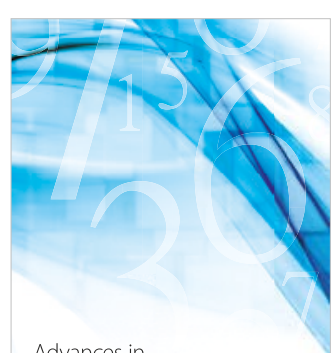

Advances in
Numerical Analysis
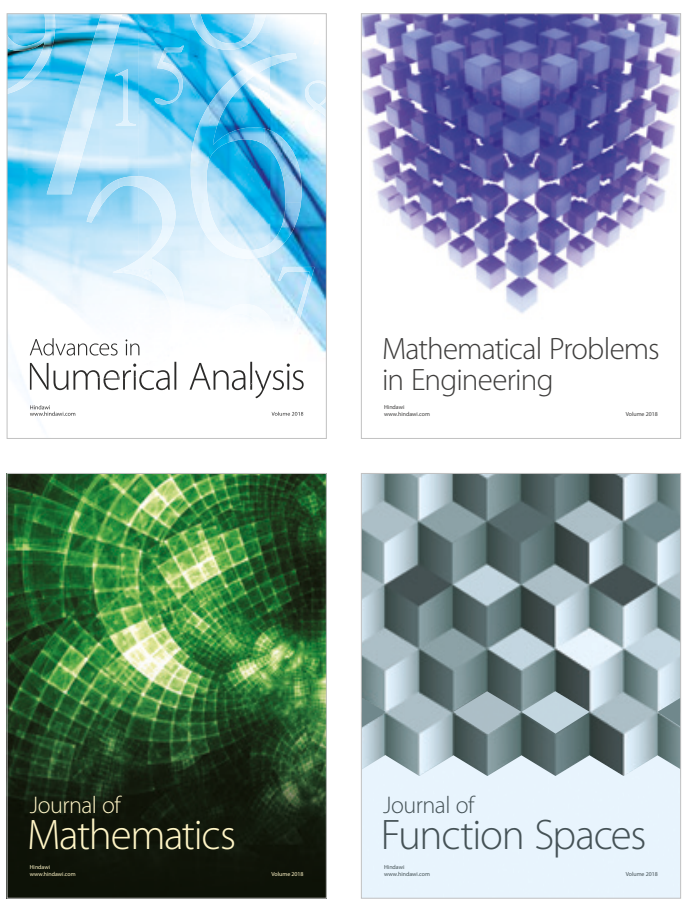

Mathematical Problems in Engineering

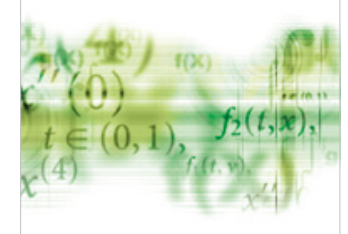

International Journal of

Differential Equations

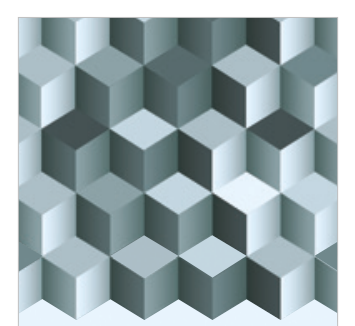

Journal of

Function Spaces
The Scientific

World Journal

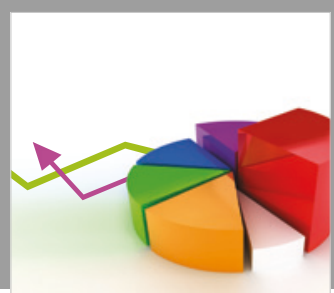

Journal of

Probability and Statistics
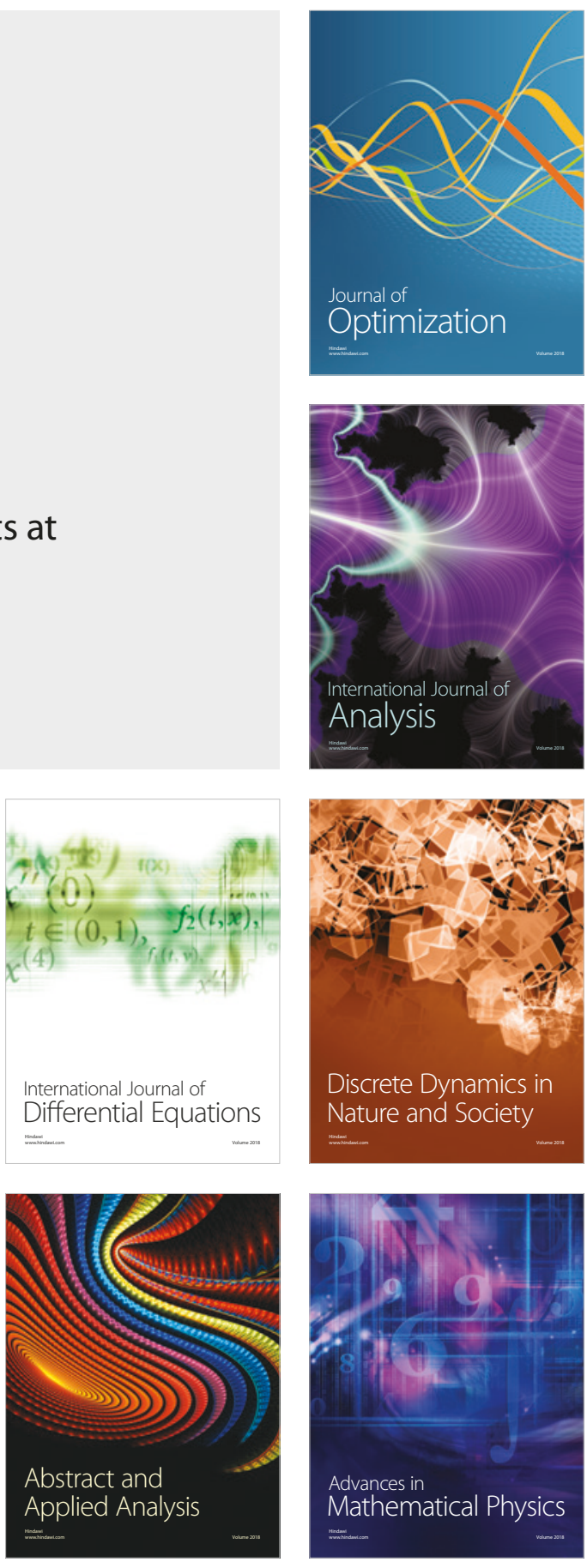